Proc. of 12th International Workshop on Positron and Positronium Chemistry, August 28-September 1, 2017, Lublin, Poland

\title{
Positronium for Antihydrogen Production in the AEGIS Experiment
}

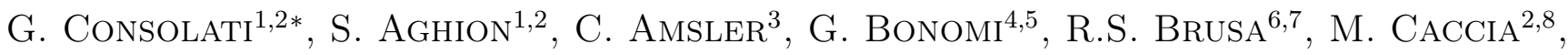
R. Caravita ${ }^{9,10,11}$, F. Castelli ${ }^{2,12}$, G. Cerchiari $^{13}$, D. Comparat $^{14}$, A. Demetrio ${ }^{15}$, L. Di Noto ${ }^{9,10}$, M. Doser ${ }^{11}$, C. Evans ${ }^{1,2}$, M. Fanì $^{9,10,11}$, R. Ferragut ${ }^{1,2}$, J. Fesel ${ }^{11}$, A. Fontana ${ }^{5}$, S. Gerber ${ }^{11}$, M. Giammarchi ${ }^{2}$, A. Gligorova ${ }^{3}$, F. Guatieri ${ }^{6,7}$, S. Haider ${ }^{11}$, A. Hinterberger ${ }^{11}$, H. Holmestad ${ }^{16}$, A. Kellerbauer ${ }^{13}$, O. Khalidova ${ }^{11}$, D. Krasnicky ${ }^{10}$, V. Lagomarsino ${ }^{9,10}$, P. Lansonneur ${ }^{17}$, P. Lebrun ${ }^{17}$, C. Malbrunot ${ }^{3,11}$, S. Mariazzi ${ }^{18}$, J. Marton ${ }^{3}$, V. Matveev $^{19,20}$, Z. Mazzotta $^{2,12}$, S.R. MÜller ${ }^{15}$, G. Nebbia ${ }^{18}$, P. Nedelec ${ }^{17}$, M. Oberthaler ${ }^{15}$, N. Pacifico ${ }^{11}$, D. Pagano ${ }^{4,5}$, L. Penasa $^{6,7}$, V. Petracei ${ }^{21}$, F. Prelz ${ }^{2}$, M. Prevedelli ${ }^{22}$, L. Ravelli ${ }^{6,7}$, B. Rienaecker ${ }^{11}$, J. Robert ${ }^{14}$, O.M. RøHne ${ }^{16}$, A. Rotondi ${ }^{5,23}$, H. SANDAKER ${ }^{16}$, R. SANTORO ${ }^{2,8}$, L. Smestad ${ }^{11,24}$, F. Sorrentino ${ }^{9,10}$, G. Testera ${ }^{10}$, I.C. Tietje ${ }^{11}$, E. Widmann ${ }^{3}$, P. YZOMBARD ${ }^{13}$, C. Zimmer ${ }^{11,13,25}$, J. ZMeSKAL ${ }^{3}$, N. ZuRLO ${ }^{5,26}$

${ }^{1}$ Politecnico di Milano, Piazza Leonardo da Vinci 32, 20133 Milano, Italy

${ }^{2}$ INFN Milano, via Celoria 16, 20133, Milano, Italy

${ }^{3}$ Stefan Meyer Institute for Subatomic Physics, Austrian Academy of Sciences, Boltzmanngasse 3, 1090 Vienna, Austria

${ }^{4}$ Department of Mechanical and Industrial Engineering, University of Brescia, via Branze 38, 25123 Brescia, Italy ${ }^{5}$ INFN Pavia, via Bassi 6, 27100 Pavia, Italy

${ }^{6}$ Department of Physics, University of Trento, via Sommarive 14, 38123 Povo, Trento, Italy

${ }^{7}$ TIFPA/INFN Trento, via Sommarive 14, 38123 Povo, Trento, Italy

${ }^{8}$ Department of Science, University of Insubria, Via Valleggio 11, 22100 Como, Italy

${ }^{9}$ Department of Physics, University of Genova, via Dodecaneso 33, 16146 Genova, Italy

${ }^{10}$ INFN Genova, via Dodecaneso 33, 16146 Genova, Italy

${ }^{11}$ Physics Department, CERN, 1211 Geneva 23, Switzerland

${ }^{12}$ Department of Physics, University of Milano, via Celoria 16, 20133 Milano, Italy

${ }^{13}$ Max Planck Institute for Nuclear Physics, Saupfercheckweg 1, 69117 Heidelberg, Germany

${ }^{14}$ Laboratoire Aime Cotton, Université Paris-Sud, ENS Paris-Saclay, CNRS, Université Paris-Saclay, 91405 Orsay Cedex, France

${ }^{15}$ Kirchhoff-Institute for Physics, Heidelberg University, Im Neuenheimer Feld 227, 69120 Heidelberg, Germany

${ }^{16}$ Department of Physics, University of Oslo, Sem Slandsvei 24, 0371 Oslo, Norway

${ }^{17}$ Institute of Nuclear Physics, CNRS/IN2p3, University of Lyon 1, 69622 Villeurbanne, France

${ }^{18}$ INFN Padova, via Marzolo 8, 35131 Padova, Italy

${ }^{19}$ Institute for Nuclear Research of the Russian Academy of Science, Moscow 117312, Russia

${ }^{20}$ Joint Institute for Nuclear Research, 141980 Dubna, Russia

${ }^{21}$ Czech Technical University, Prague, Brehov 7, 11519 Prague 1, Czech Republic

${ }^{22}$ University of Bologna, Viale Berti Pichat 6/2, 40126 Bologna, Italy

${ }^{23}$ Department of Physics, University of Pavia, via Bassi 6, 27100 Pavia, Italy

${ }^{24}$ The Research Council of Norway, P.O. Box 564, NO-1327 Lysaker, Norway

${ }^{25}$ Department of Physics, Heidelberg University, Im Neuenheimer Feld 226, 69120 Heidelberg, Germany

${ }^{26}$ Department of Civil, Environmental, Architectural Engineering and Mathematics, University of Brescia, via Branze 43, 25123 Brescia, Italy

The primary goal of the Antihydrogen Experiment: Gravity, Interferometry, Spectroscopy (AEGIS) collaboration is to measure for the first time precisely the gravitational acceleration of antihydrogen, $\overline{\mathrm{H}}$, a fundamental issue of contemporary physics, using a beam of antiatoms. Indeed, although indirect arguments have been raised against a different acceleration of antimatter with respect to matter, nevertheless some attempts to formulate quantum theories of gravity, or to unify gravity with the other forces, consider the possibility of a non-identical gravitational 
interaction between matter and antimatter. We plan to generate $\overline{\mathrm{H}}$ through a charge-exchange reaction between excited Ps and antiprotons coming from the Antiproton Decelerator facility at CERN. It offers the advantage to produce sufficiently cold antihydrogen to make feasible a measurement of gravitational acceleration with reasonable uncertainty (of the order of a few percent). Since the cross-section of the above reaction increases with $n^{4}, n$ being the principal quantum number of Ps, it is essential to generate Ps in a highly excited (Rydberg) state. This will occur by means of two laser excitations of Ps emitted from a nanoporous silica target: a first UV laser (at $205 \mathrm{~nm}$ ) will bring Ps from the ground to the $n=3$ state; a second laser pulse (tunable in the range 1650-1700 nm) will further excite Ps to the Rydberg state.

DOI: 10.12693/APhysPolA.132.1443

PACS/topics: 04.80.Cc, 07.77.-n, 36.10.Dr, 78.70.Bj

\section{Introduction}

Antimatter is a hot topic in contemporary physics, owing to its important role in our understanding of fundamental interactions. Precision spectroscopy measurements on antimatter are considered an important test of the validity of the CPT theorem [1]. In fact, a possible violation of the theorem is envisaged in some extensions of the standard model [2]. Another issue concerns the gravitational interaction on antimatter. The weak equivalence principle (WEP) postulates that the trajectory and the velocity of a body falling in an external gravitational field are not affected by its composition, but depends only on its initial position and velocity. Many careful tests verified the WEP at $10^{-13}$ level for ordinary matter [3]. Indirect arguments have been raised against a different acceleration of antimatter with respect to matter; however, some attempts to formulate quantum theories of gravity, or to unify gravity with the other forces [4] consider the possibility of a non-identical gravitational interaction between matter and antimatter.

Measurements with charged antimatter are very complicated, owing to the overwhelming effect of residual electromagnetic forces [5]. Therefore, neutral antimatter, that is, e.g. antiatoms, is of great interest to investigate the gravitational interaction. The only antiatom which will be most likely possible to investigate in the next future is antihydrogen, $\overline{\mathrm{H}}$. In particular, a measurement of the gravitational acceleration $\boldsymbol{g}$ on $\overline{\mathrm{H}}$, even with a few percent precision, would be scientifically relevant, as it would represent the first direct measurement of the gravitational interaction between matter and antimatter [6].

The first production of $\overline{\mathrm{H}}$ occurred in 1996 at CERN [7] and two years later at Fermilab [8]; confinement of antihydrogen was however impossible, the energies being of the order of a few GeV. The ATHENA [9] and the ATRAP [10] experiments in 2002 obtained for the first time cold $\overline{\mathrm{H}}$. Nowadays, four experiments (ATRAP, ALPHA, ASACUSA and AEGIS) are currently running at CERN, with the aim to produce antihydrogen with more advanced features (e.g. trappable $\overline{\mathrm{H}}$ for spectroscopy studies, or $\overline{\mathrm{H}}$ beams), by using the antiproton deceler-

\footnotetext{
* corresponding author; e-mail: giovanni. consolati@polimi.it
}

ator (AD) facility, which supplies about $3 \times 10^{7}$ antiprotons $(\overline{\mathrm{p}})$ - with kinetic energy of $5.3 \mathrm{MeV}$ - in the form of pulses of about $200 \mathrm{~ns}$ and at a repetition rate of $0.009 \mathrm{~s}^{-1}$. A fifth experiment, GBAR, has been approved in 2012 and the different parts of the apparatus are under construction.

In particular, the first scientific goal of the AEGIS collaboration is to measure the gravitational acceleration $\boldsymbol{g}$ of a beam of cold $\overline{\mathrm{H}}$ in the Earth gravitational field with one percent order precision [11]. A primary ingredient of the experiment is positronium, Ps. Indeed, the antiatoms will be produced by means of a charge exchange reaction among excited Ps atoms and cooled antiprotons.

In the present paper we give an overview of the AEGIS experiment and we describe its current status.

\section{Overview of the AEGIS experiment}

The essential steps to produce cold $\overline{\mathrm{H}}$ as well as to measure its gravitational acceleration can be summarized under the following items: (a) trapping and accumulation of antiprotons coming from the AD facility, (b) $\overline{\mathrm{p}}$ cooling to cryogenic temperatures, (c) production of positrons and their storage into an accumulator, (d) production and emission into vacuum of cold $(<150 \mathrm{~K})$ Ps formed from impinging a positron bunch on a suitable nanoporous target, (e) Ps excitation in a Rydberg state $\left(\mathrm{Ps}^{*}\right)$ by means of two suitable laser pulses, (f) formation of cold Rydberg $\overline{\mathrm{H}}\left(\bar{H}^{*}\right)$ from a charge exchange reaction between antiprotons and $\mathrm{Ps}^{*}$, (g) extraction of $\bar{H}^{*}$ atoms by means of inhomogeneous electric fields (the Stark acceleration technique), (h) measurement of antihydrogen vertical deflection due to gravitational acceleration using a moiré deflectometer coupled to a position sensitive detector.

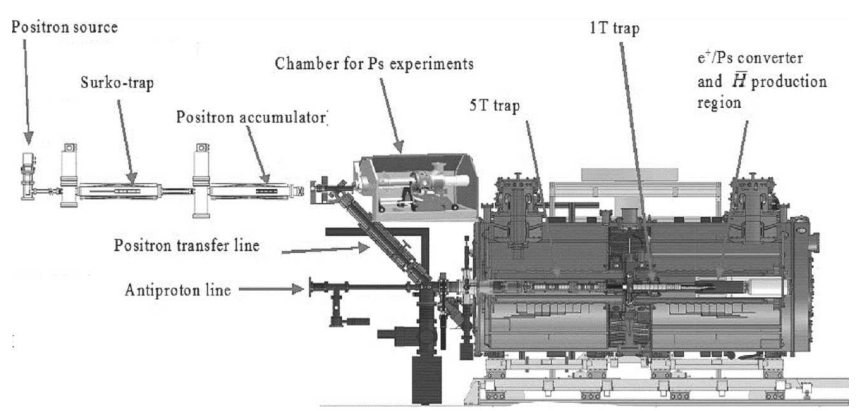

Fig. 1. Sketch of the AEGIS apparatus. 
The core of the AEGIS apparatus consists of the positron system and a cryogenic system in ultra high vacuum composed by two magnets ( $5 \mathrm{~T}$ and $1 \mathrm{~T}$ ), where a complex system of traps is located (Fig. 1). Antiprotons delivered from the AD pass through a degrader (a stack of $\mathrm{Al}$ foils, with variable thicknesses) and are caught in a Penning-Malmberg trap placed in the $5 \mathrm{~T}$ magnet.

About $3 \times 10^{5} \overline{\mathrm{p}}$ are trapped for each antiproton shot, by using a suitably optimized trapping potential. By Coulombian collisions, $\overline{\mathrm{p}}$ transfer their energy to about $10^{8}$ electrons (previously injected in the trap) which cool down to cryogenic temperatures by irradiating within a few ms (sympathetic cooling). The time needed for the $\overline{\mathrm{p}}$ temperature to stabilize is typically of the order of one minute. In this time interval a $\bar{p}$ cooling efficiency of $90 \%$ is obtained.

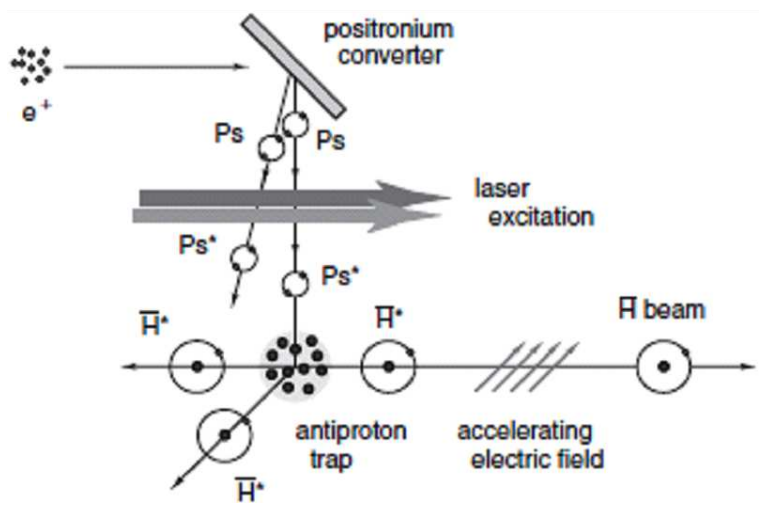

Fig. 2. Scheme of the method used by the AEGIS collaboration to produce a beam of cold antihydrogen.

Then, $\overline{\mathrm{p}}$ are compressed by applying an oscillating potential on an electrode segmented azimuthally ("Rotating Wall" technique) and are finally sent toward a trap located in the $1 \mathrm{~T}$ region, where they react with $\mathrm{Ps}^{*}$ to produce $\overline{\mathrm{H}}$ (Fig. 2) according to the following chargeexchange reaction:

$$
\mathrm{Ps}^{*}+\overline{\mathrm{p}} \rightarrow \overline{\mathrm{H}}+\mathrm{e}^{-} \text {. }
$$

The cross-section of the reaction (1) increases with $n^{4}$, where $n$ is the principal quantum number of Ps; this is the reason why it is mandatory to produce Ps in a Rydberg state. This will occur by means of two laser excitations of Ps emitted in a vacuum from a porous target: a first UV laser (at $205 \mathrm{~nm}$ ) will bring Ps from the ground to the $n=3$ state; a second, tunable IR laser pulse will excite Ps to the final Rydberg state [12].

As a consequence of the reaction (1) $\overline{\mathrm{H}}$ will be formed in a Rydberg state, hence, with a large electric dipole moment. This gives the possibility to accelerate $\overline{\mathrm{H}}$ in the presence of an inhomogeneous electric field. This technique, already tested with hydrogen [13], will allow the antiatoms to be driven along the gravity measurement module. Their time of flight $T$ will be measured from the time span between the Stark acceleration time and a timing detector positioned at the end of the gravity module: a moiré deflectometer. This last consists of two identical gratings, perpendicular to the trajectory of $\overline{\mathrm{H}}$, with pitch $d$ and placed at a distance $L$ from each other [14]. Among the particles traversing the two gratings only those ones having well defined trajectories are selected (Fig. 3) and produce a fringe pattern in a third plane, located at the same distance $L$ from the second grating.

Among the possible antihydrogen detectors the best candidates seem silicon detectors with thin active volume [15], which helps to localize spatially the annihilation event through the detection of highly ionizing fragments. Time resolutions in the nanoseconds range can be achieved. In particular, the Timepix [16], initially developed for medical imaging applications, is able to determine with $25 \mu \mathrm{m}$ spatial resolution the position of an antiproton annihilation with more than $50 \%$ tagging efficiency. Also nuclear emulsions were tested [17]. They consist of plates of plastic or glass covered with $\mathrm{AgBr}(\mathrm{I})$ crystals distributed homogeneously in a gelatin substrate. The emulsion plate can be used as tracker device to determine the antihydrogen annihilation vertices with very high precision: resolutions of less than $2 \mu \mathrm{m}$ root mean square (r.m.s.) have been obtained with antiprotons [18]. Unfortunately, nuclear emulsions do not work in the cryogenic and ultra high vacuum environment required by the AEGIS apparatus and require frequent opening of the vacuum chamber to be replaced and processed. This makes troublesome their use in our experiment. Furthermore, timing is not possible with nuclear emulsions and they should operate in any case in combination with a detector with good time resolution.

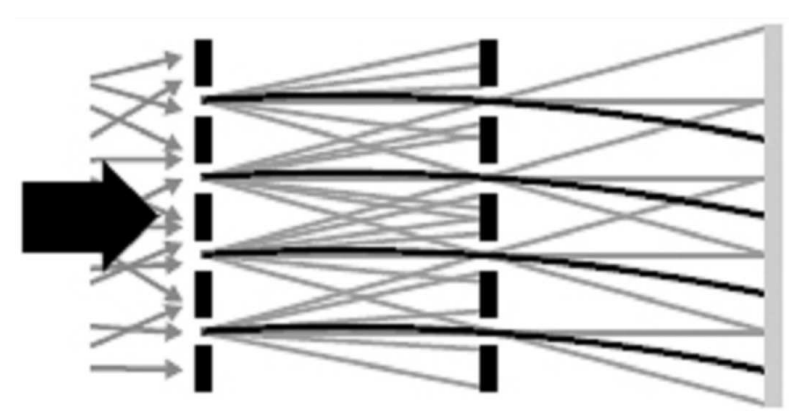

Fig. 3. The moiré deflectometer. The trajectories of undisturbed particles (grey lines) are modified by the presence of a force (black lines) and a shift of the fringe pattern occurs. In the case of gravity the shift $\delta$ depends on both time of flight $T$ and $\boldsymbol{g}: \delta=\boldsymbol{g} T^{2} . \boldsymbol{g}$ can be obtained by fitting the shift versus $T$ on an event-byevent basis. The data will be recorded by means of a suitable detector placed in the third plane.

\section{Positron confinement, Ps formation and excitation}

Positrons emitted by a ${ }^{22} \mathrm{Na}$ radioactive source (about $50 \mathrm{mCi}$ ) are slowed down by means of a solid Ne moderator [19], captured in a two-stage Surko buffer trap [20] and stored in an accumulator. Here bunches of some $10^{7} \mathrm{e}^{+}$are released with a longitudinal velocity corresponding to an energy of $300 \mathrm{eV}$, magnetically trans- 
ported in a $0.14 \mathrm{~T}$ field and injected into the PenningMalmberg trap in a $5 \mathrm{~T}$ magnetic field.

Our measurements show that positrons trapped in a 50-100 eV potential well are cooled down by cyclotron radiation with almost $100 \%$ efficiency for more than $30 \mathrm{~min}$. The long lifetime of the positrons in the trap makes possible to accumulate more than $2 \times 10^{8}$ positrons.

Ps formation, emission into vacuum and excitation in the Rydberg states have been demonstrated by us, using a "chamber for Ps experiments" [21] in line with the Surko trap and the accumulator, as shown in Fig. 1. A bunch of positrons impinging on a Si target with oriented oxidized nanochannels $[22,23]$ produced copious Ps emission in vacuum. Ps excitation was measured by means of the single shot positron annihilation lifetime spectroscopy (SSPALS) technique [24]. The lifetime spectra from the single gamma ray shots were acquired with a $\mathrm{PbWO}_{4}$ scintillator coupled to a Hamamatsu R11265-100 photomultiplier tube. A laser system to perform the two-step Ps excitation: $1^{3} S \rightarrow 3^{3} \mathrm{P}, 3^{3} \mathrm{P} \rightarrow$ the Rydberg state was specifically designed and installed [12]. In a first experiment, a UV laser pulse $(\lambda=205 \mathrm{~nm}$, energy $54 \mu \mathrm{J}$, pulse width $1.5 \mathrm{~ns}$ ) excited Ps from ground to $n=3$ state; simultaneously, an IR laser $(\lambda=1064 \mathrm{~nm}$, energy $50 \mathrm{~mJ}$, pulse width about $10 \mathrm{~ns}$ ) was shot to ionize the excited Ps, in the presence of a $600 \mathrm{~V} / \mathrm{cm}$ electric field, to demonstrate Ps excitation. Afterwards, an IR laser (tuneable wavelength in the 1650 to $1720 \mathrm{~nm}$ range, energy $=1 \mathrm{~mJ}$, pulse width about $4 \mathrm{~ns}$ ) was pulsed at the same time with the UV laser to excite Ps from $n=3$ to the Rydberg levels.

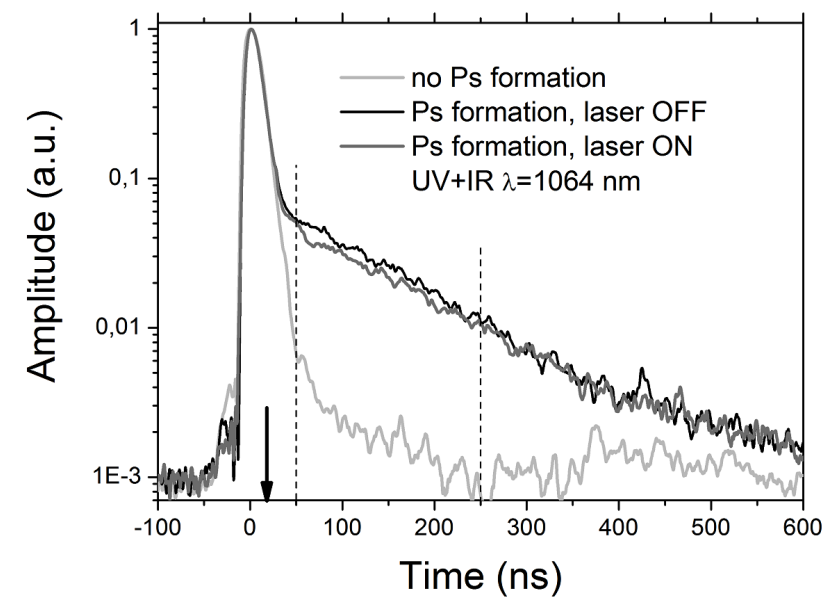

Fig. 4. SSPALS spectra. Light grey line: background; black: Ps in vacuum, laser OFF; dark grey: UV + IR lasers ON (205.05 + $1064 \mathrm{~nm})$. Arrow: time when the laser is shot on the Ps cloud (16 ns after the prompt peak). The area between 50 and $250 \mathrm{~ns}$ from the prompt peak (vertical dashed lines) was used to evaluate parameter $S$ for $n=3$. Reproduced from Ref. [25].

Results are shown in Figures 4 to 7 [25]. Ps decaying in vacuum is represented by the black lines after the annihilation prompt peak. The dark grey curve in Fig. 4

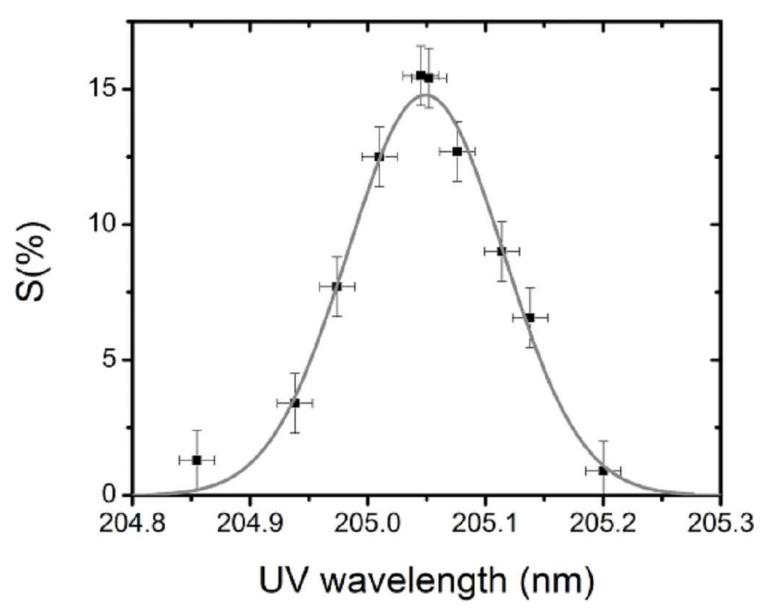

Fig. 5. Linewidth of the $1^{3} S \rightarrow 3^{3} P$ Ps excitation as obtained by scanning the UV laser wavelength. Reproduced from Ref. [25].

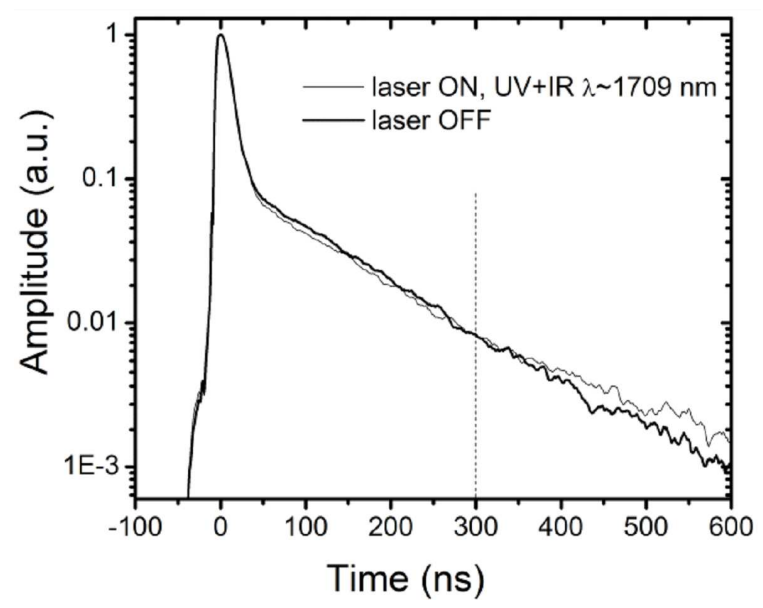

Fig. 6. SSPALS spectra of Ps in vacuum. Black curve: $\mathrm{UV}+$ IR lasers OFF; grey curve: laser UV + IR ON $(205.05+1709 \mathrm{~nm})$. Area between $300 \mathrm{~ns}$ (vertical dashed line) and $600 \mathrm{~ns}$ from the prompt peak has been considered for evaluation of the $S$ parameter for the Rydberg levels. Reproduced from Ref. [25].

shows the decrease of Ps due to Ps ionization induced by the UV + IR $(\lambda=1064 \mathrm{~nm})$ lasers simultaneously sent on the Ps cloud. Parameter $S=\left(f_{\text {off }}-f_{\text {on }}\right) / f_{\text {off }}$ quantifies the decrease, where $f_{\text {off }}$ and $f_{\text {on }}$ are the areas of the SSPAL spectra between 50 and 250 ns when the laser is turned off and on, respectively.

The $1^{3} S \rightarrow 3^{3} \mathrm{P}$ excitation linewidth as obtained by measuring $S \%$ as a function of the UV wavelength is shown in Fig. 5.

Ps excited in the Rydberg states has a lifetime of the order of microseconds, therefore it can reach the walls of the vacuum chamber. Indeed, a decrease of annihilations is observed in the SSPALS spectrum after the prompt peak due to the $\mathrm{Ps}^{*}$ formation (grey curve be- 
fore $300 \mathrm{~ns}$ in Fig. 6). Conversely, annihilations increase when $\mathrm{Ps}^{*}$ starts reaching the chamber walls (grey curve after 300 ns, Fig. 6).

Figure 7 shows the negative of the $S$ parameter versus the wavelength of the IR laser, the UV laser being kept constant at $\lambda=205.05 \mathrm{~nm}(n=3$ resonance). This scanning allowed us to resolve the $n=15$ Rydberg line; the $n=16-17$ lines cannot be distinctly resolved owing to the excessive broadening.

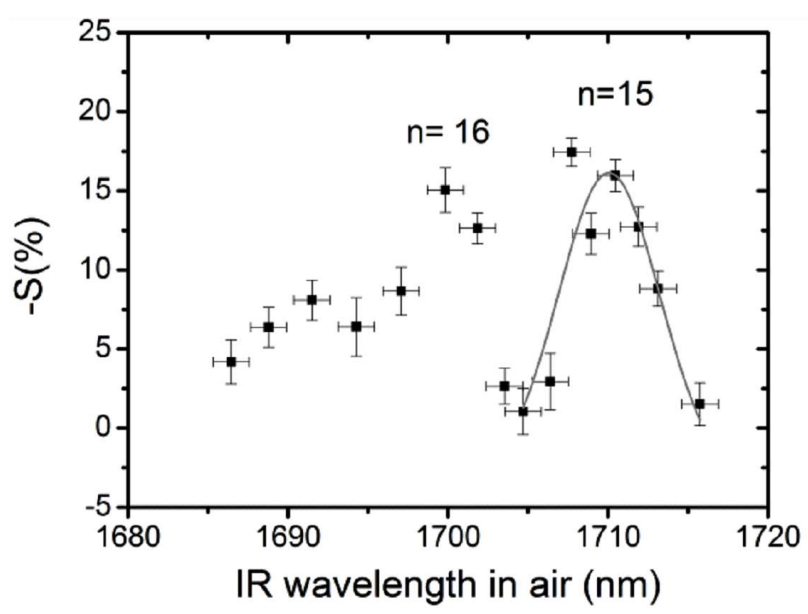

Fig. 7. Scan of the $S$ parameter versus the IR wavelength. The peak associated to the transitions to $n=15$ is fitted with a Gaussian. For $n>16$, lines are not clearly separated due to the wide broadening. Reproduced from Ref. [25].

\section{Ps converters in transmission geometry}

In our experiment Ps is presently formed in the usual reflection geometry, that is, it is emitted from the same surface of the converter on which positrons are implanted. Recently, a transmission geometry has been envisaged by taking advantage from the development of thin mesostructured silica film targets [26-28]. Presently, transmission converters are not competitive as far as Ps yield and cooling efficiency are concerned. Nevertheless, they could be advantageous for antihydrogen production. Indeed, in transmission geometry the $\overline{\mathrm{p}} / \mathrm{Ps}$ charge exchange reaction is expected to increase in efficiency due to the possibility to move the target nearer to the antiproton cloud, granting higher geometrical overlap between antiprotons and $o$-Ps.

Problems could arise, owing to the limited thickness of the target, which would allow a fraction of positrons partially thermalized to emerge together with o-Ps. Also secondary electrons produced by interaction between positrons and the target would be emitted. This could heat the antiproton cloud, with corresponding increase of the temperature of the antihydrogen produced.

By using three different techniques (SSPALS, time-offlight and imaging of particles impinging a microchannel plate), we recently characterized a transmission
positron/Ps converter for its possible application in antihydrogen production [29]. It consists of an ultraporous mesostructured silica film (density about $0.4 \mathrm{~g} / \mathrm{cm}^{3}$ ) evaporated onto a $20 \mathrm{~nm}$ carbon foil. The target thickness is about $750 \mathrm{~nm}$. Positrons were implanted at two different energies: 3.3 and $4.5 \mathrm{keV}$. We found a fraction of Ps forward-emitted in vacuum equal to 7 and $3 \%$, respectively, to be compared to 8 and $4 \%$ in reflection geometry. The decrease of Ps fraction is due to the smaller number of positrons stopped in the target when their energy is increased.

Positrons crossing the target and secondary electrons produced by positrons slowing down in the target were imaged by means of a microchannel plate/phosphor screen assembly connected to a charge-coupled device camera placed $0.8 \mathrm{~cm}$ downstream the $\mathrm{e}^{+} / \mathrm{Ps}$ converter. We estimated that at least $10 \%$ of the implanted positrons at $3.3 \mathrm{keV}$ are forward emitted with a maximum kinetic energy of $1.2 \mathrm{keV}$, and about $0.1-0.2$ secondary electrons are emitted per implanted positron. In order to avoid interactions of these charged particles with the antiproton cloud, two grids with high transmission coefficients could be placed downstream the converter, very near its surface. The first grid, closest to the target, positively polarized would reflect the re-emitted positrons. The second grid, immediately following and set at a negative potential would repel the secondary electrons. Moreover, this should be at the same potential as on the first electrode of the trap, in order to have a region with no electric field downstream this grid. This way ground state $o$-Ps could cross the two grids without being affected by the electric field and subsequently be excited to the Rydberg states in a field-free region.

\section{Proof of principle of the moiré deflectometer}

The design of the moiré deflectometer will take advantage of the experience gained on a compact prototype device, which has worked with antiprotons [30]. It is formed by two parallel gratings (distance $25 \mathrm{~mm}$ from each other) and an emulsion detector (resolution $2 \mu \mathrm{m}$ ) placed $25 \mathrm{~mm}$ from the second grating. Dimensions of the slits in the $100 \mu \mathrm{m}$ thick silicon grating (12 $\mu \mathrm{m}$ width and a $40 \mu \mathrm{m}$ periodicity) granted a classical regime when compared to the de Broglie wavelength of the antiprotons $\left(8.8 \times 10^{-8} \mu \mathrm{m}\right)$.

A beam of particles (mean energy $106 \mathrm{keV}$ ) passing through the deflectometer produces a fringe pattern on the detector; the presence of a force shifts such a pattern. To infer the force, a comparison with a near-field interference pattern produced by light has been carried out. An additional transmission grating in direct contact with the emulsion was illuminated simultaneously with the moiré deflectometer, with antiprotons as well as with light. This provided a reference for alignment, since the pattern behind the contact grating cannot show any dependence on a force. The results showed an upward shift in the moiré pattern with respect to the interference pattern due to the light, given by $\Delta y=F \tau^{2} / m$, 
where $F$ is the force perpendicular to the slits, $\tau$ is the time of flight between the two gratings and $m$ is the antiproton mass. The observed shift, $\Delta y=9.8( \pm 0.9) \mu \mathrm{m}$ (stat.) $( \pm 6.4) \mu \mathrm{m}$ (syst.) (Fig. 8), corresponds to a force acting on $\overline{\mathrm{p}}$ of $530( \pm 50)$ aN (stat.) ( \pm 350$)$ aN (syst.).

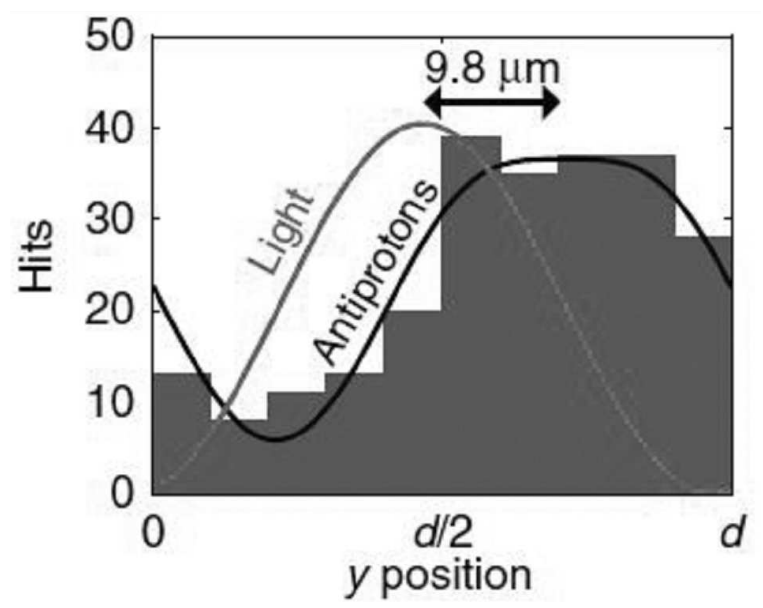

Fig. 8. Light and antiproton patterns showing the observed shift of antiprotons through the moiré deflectometer. Reproduced from Ref. [30].

Since the moiré deflectometer and the emulsion detector were mounted at the end of the $1 \mathrm{~T}$ magnet (Fig. 1) in a dedicated vacuum chamber, such a force was identified with a Lorentz force due to the magnetic field. A magnetic induction $B$ of about 7.4 Gs was estimated, which is good agreement with the magnetic induction around $10 \mathrm{Gs}$, measured at the position of the deflectometer and perpendicular to the direction of $\bar{p}$. These results are essential for the final design of the deflectometer working with $\overline{\mathrm{H}}$. Indeed, the fringe pattern of $\overline{\mathrm{H}}$ due to gravity is expected to be comparable to the one observed in the case of antiprotons. It is true that the gravitational force acting on $\overline{\mathrm{H}}$ is expected to be about ten orders of magnitude smaller than the sensitivity level reached with the prototype device, but the resolution of the setup will be improved by scaling up the deflectometer (distance between gratings about $1 \mathrm{~m}$ ). Furthermore, the velocity of $\overline{\mathrm{H}}$ should be four orders of magnitude smaller than the velocity of the $\bar{p}$ used in the experiment described above. As a result, an improvement of about eleven orders of magnitude sensitivity can be expected with respect to the prototype.

\section{Conclusions}

The challenging first goal of the AEGIS collaboration, the measurement of antihydrogen gravitational acceleration with a few percent uncertainty, requires expertise in various fields: manipulation, storage and cooling of antiprotons; production of suitable bunches of positrons and their control to impinge a nanoporous target for generation and emission of cold Ps in vacuum; the Rydberg excitation of $\mathrm{Ps}$ and control of the corresponding reaction with antiprotons; manipulation of antihydrogen to be directed towards the final stage, the moiré deflectometer. After completing the commissioning of the apparatus, we are now able to capture about $3 \times 10^{5}$ antiprotons and to cool them with $90 \%$ efficiency. More than $2 \times 10^{7}$ positrons can actually be stored in the $5 \mathrm{~T}$ magnet for tens of minutes without significant losses. After implanting positron bunches in a nanoporous silicon target, Ps atoms were emitted in vacuum in a chamber designed for Ps experiments and the Rydberg excitation was demonstrated by using a double UV-IR laser system. Therefore, we have at hand all the ingredients to produce antihydrogen by means of the charge-exchange reaction. Finally, we proved the feasibility of a moiré deflectometer for the gravitational fall of antihydrogen by setting up a prototype able to measure an equivalent (magnetic) deflection of an antiproton beam.

Meanwhile, we are actively exploring the production of Ps in transmission geometry, which may offer various advantages with respect to the traditional reflection mode.

Current antihydrogen experiments at CERN are limited by the trapping efficiency of antiprotons, coming from $\mathrm{AD}$ at $5.3 \mathrm{MeV}$, which have to be slowed down before being manipulated. In the next future Extra Low Energy Antiproton Ring (ELENA) [31], a further deceleration step, will start operation, lowering the energy of the available antiprotons to $100 \mathrm{keV}$. This will increase the $\bar{p}$ catching efficiency by up two orders of magnitude. Then, it will be possible to run several experiments in parallel (presently the beam time is shared among the various groups) with much more antiprotons available, opening new perspectives in cold antimatter physics.

\section{References}

[1] G. Lüders, Ann. Phys. 2, 1 (1957).

[2] D. Colladay, V.A. Kostelecky, Phys. Rev. D 55, 6760 (1997).

[3] E.G. Adelberger, J.H. Gundlach, B.R. Heckel, S. Hoedl, S. Schlamminger, Prog. Part. Nucl. Phys. 62, 102 (2009).

[4] J. Barrow, R. Scherrer, Phys. Rev. D 70, 103515 (2004).

[5] T.W. Darling, F. Rossi, G.I. Opat, G.F. Moorhead, Rev. Mod. Phys. 64, 237 (1992).

[6] M. Doser et al. (AEGIS collaboration), Class. Quantum Grav. 29, 184009 (2012).

[7] G. Baur, G. Boero, S. Brauksiepe, A. Buzzo, W. Eyrich, G. Geyer, D. Grzonka, J. Hauffe, K. Kilian, M. LoVetere, M. Macri, M. Moosburger, R. Nellen, W. Oelert, S. Passaggio, A. Pozzo, K. Röhrich, K. Sachs, G. Schepers, T. Sefzick, R.S. Simon, R. Stratmann, F. Stinzing, M. Wolke, Phys. Lett. B 368, 251 (1996).

[8] G. Blanford, D.C. Christian, K. Gollwitzer, M. Mandelkern, C.T. Munger, J. Schultz, G. Zioulas, Phys. Rev. Lett. 80, 3037 (1998).

[9] M. Amoretti, ATHENA collaboration, Nature 419, 456 (2002). 
[10] G. Gabrielse, ATRAP collaboration, Phys. Rev. Lett. 89, 213401 (2002).

[11] A. Kellerbauer, AEGIS collaboration, Nucl. Instrum. Methods Phys. Res. B 266, 351 (2008).

[12] S. Cialdi, I. Boscolo, F. Castelli, F. Villa, G. Ferrari, M.G. Giammarchi, Nucl. Instrum. Methods Phys. Res. B 269, 1527 (2011).

[13] E. Vliegen, S.D. Hogan, H. Schmutz, F. Merkt, Phys. Rev. A 76, 023405 (2007).

[14] M.K. Oberthaler, S. Bennet, E.M. Rasel, J. Schniedmayer, A. Zeilinger, Phys. Rev. A 54, 3165 (1996).

[15] N. Pacifico et al. (AEGIS collaboration), Nucl. Instrum. Methods Phys. Res. A 765, 161 (2014).

[16] T. Poikela, J. Plosila, T. Westerlund, M. Campbell, M. De Gaspari, X. Llopart, V. Gromov, R. Kluit, M. van Beuzekom, F. Zappon, V. Zivkovic, C. Brezina, K. Desch, Y. Fu, A. Kruth, JINST 9, C05013 (2014).

[17] M. Kimura, AEGIS collaboration, Nucl. Instrum. Methods Phys. Res. A 732, 325 (2013).

[18] S. Aghion, AEGIS collaboration, JINST 8, P08013 (2013).

[19] A.P. Mills, Jr., E.M. Gullikson, Appl. Phys. Lett. 49, 1121 (1986)

[20] J.R. Danielson, D.H.E. Dubin, R.G. Greaves, C.M. Surko, Rev. Mod. Phys. 87, 247 (2015).
[21] S. Aghion, AEGIS collaboration, Nucl. Instrum. Methods Phys. Res. B 362, 86 (2015).

[22] S. Mariazzi, P. Bettotti, S. Larcheri, L. Toniutti, R.S. Brusa, Phys. Rev. B 81, 235418 (2010).

[23] S. Mariazzi, P. Bettotti, R.S. Brusa, Phys. Rev. Lett. 104, 243401 (2010).

[24] D.B. Cassidy, S.H.M. Deng, H.K.M. Tanaka, A.P. Mills, Jr., Appl. Phys. Lett. 88, 194105 (2006).

[25] S. Aghion et al. (AEGIS collaboration), Phys. Rev. A 94, 012507 (2016)

[26] S.L. Andersen, R.R. Johansen, J.B. Overgaard, J.K. Mortensen, K.K. Andersen, H.D. Thomsen, M.D. Lund, J. Chevallier, H. Knudsen, U.I. Uggerhj, Eur. Phys. J. D 68, 124 (2014).

[27] S.L. Andersen, D.B. Cassidy, J. Chevallier, B.S. Cooper, A. Deller, T.E. Wall, U.I. Uggerhj, J. Phys. B At. Mol. Opt. Phys. 48, 204003 (2015).

[28] S.L. Andersen, Ph.D. Thesis, Department of Physics and Astronomy, Aarhus University, 2015.

[29] S. Aghion, AEGIS collaboration, Nucl. Instrum. Methods Phys. Res. B 407, 55 (2017).

[30] S. Aghion, AEGIS collaboration, Nature Commun. 5, 4538 (2014)

[31] W. Oelert, Acta Phys. Pol. B 46, 181 (2015). 